\title{
Optimierte Polymer-Rohrwerkstoffe für effiziente Drainagesysteme in Tunnelbauwerken - PolyDrain
}

\author{
Florian Arbeiter ${ }^{1}$, Stefanie Eichinger ${ }^{2}$, Gisbert Rieß ${ }^{3}$, Tobias Schachinger ${ }^{4}$, Ronny Boch ${ }^{2}$, Robert Wenighofer ${ }^{5}$, \\ Robert Galler ${ }^{5}$, Andreas Hausberger ${ }^{6}$, Elmar Strobl7, Michael Stur ${ }^{8}$, Florian Saliger ${ }^{4}$, Michael Steiner ${ }^{9}$, \\ Martin Dietzel ${ }^{2}$ und Gerald Pinter ${ }^{1}$
}

\begin{abstract}
${ }^{1}$ Lehrstuhl für Werkstoffkunde und Prüfung der Kunststoffe, Montanuniversität Leoben, Leoben, Österreich ${ }^{2}$ Institut für Angewandte Geowissenschaften, Technische Universität Graz, Graz, Österreich ${ }^{3}$ Lehrstuhl für Chemie der Kunststoffe, Montanuniversität Leoben, Leoben, Österreich ${ }^{4}$ Österreichische Bundesbahnen ÖBB Infrastruktur AG, Wien, Österreich ${ }^{5}$ Lehrstuhl für Subsurface Engineering, Montanuniversität Leoben, Leoben, Österreich ${ }^{6}$ Polymer Competence Center Leoben $\mathrm{GmbH}$, Leoben, Österreich 'Ingenieurbüro Strobl, Strobl, Österreich ${ }^{8}$ Institut für Angewandte Geologie, Universität für Bodenkultur Wien, Wien, Österreich ${ }^{9}$ Autobahnen- und Schnellstraßen-Finanzierungs-Aktiengesellschaft ASFINAG, Wien, Österreich
\end{abstract}

Eingegangen 15. Oktober 2019; angenommen 18. Oktober 2019; online publiziert 18. November 2019

\begin{abstract}
Zusammenfassung: Ausgehend von der Problematik aufwändiger Instandhaltungsarbeiten für die Funktionstüchtigkeit von Drainagerohren in Tunnelbauwerken wurde das interdisziplinäre Forschungsprojekt „PolyDrain“ initiiert. Im Rahmen des Forschungsprojekts werden diesbezüglich kritische Bauwerke in Österreich erhoben und vielversprechende Methoden zur Verringerung von zumeist karbonatischen Versinterungen in Drainagerohren untersucht. Durch gezielte Materialmodifikationen der Drainagerohre soll der Instandhaltungsaufwand zukünftiger oder zu sanierender Tunnelbauwerke reduziert werden, indem Reinigungsintervalle gestreckt und/oder Reinigungen rascher durchgeführt werden können. Der gewählte Ansatz umfasst die maßgeschneiderte Modifizierung von KunststoffDrainagerohrmaterialien. Dadurch ist es möglich, mechanisch widerstandsfähigere Rohre zu erzeugen und/oder das Versinterungsverhalten im Drainagerohr, inklusive der Drainageschlitze, zu reduzieren. Bevor eine Umsetzung im großtechnischen Maßstab möglich ist, ist es allerdings wichtig, nicht nur die kurzzeitige Wirksamkeit - im Projekt auch im Labor - zu untersuchen.
\end{abstract}

Schlüsselwörter: Drainagerohr, Tunnelbau, Versinterung, Materialentwicklung

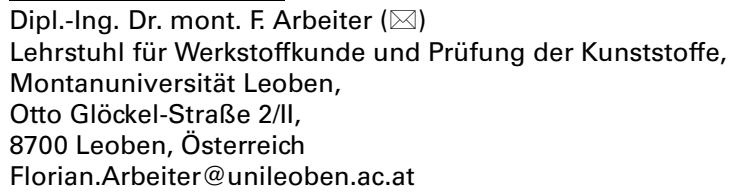

Optimized Polymer Pipe Materials for Efficient Drainage Systems in Tunneling Applications-PolyDrain

Abstract: Keeping tunnel drainage systems operational requires to deploy less expensive and time-consuming maintenance procedures. Therefore, the inter-disciplinary research project "PolyDrain" was initiated. This project comprises conducting a survey of critical tunnels in Austria as well as developing methods to decrease scale deposits in drainage pipes. The chosen approach is based on modifying the polymer matrix of drainage pipes by adding active fillers to obtain a final composite material. Accordingly, the durability of pipes can be increased and/or the precipitation can be reduced in the drainage, including areas of drainage slits. However, before these findings can be applied in large scale reality, further long-term evaluations-also in the lab-are necessary.

Keywords: Drainage pipes, Tunneling, Scale deposits, Material development

\section{Einleitung - Drainagerohre im Tunnelbau}

Drainagesysteme dienen in druckwasserentlasteten Tunnelbauwerken der permanenten konzentrierten Ableitung 
des anfallenden Grundwassers. Diese Maßnahme ist notwendig, um bei höheren Überlagerungen durch den Grundwasserspiegel den Tunnel überhaupt errichten zu können bzw. dient dazu, eine technisch und wirtschaftlich zweckmäßige Konstruktion zu ermöglichen. Bis zur zweiten Hälfte des 20. Jahrhunderts wurden für die Wasserableitung meistens gemauerte Sohlkanäle verwendet. In weiterer Folge werden bis heute Rohre aus verschiedenen Materialien für Drainage und Wasserableitungen verwendet. Seit den 90er Jahren werden ausschließlich Rohre aus thermoplastischen Kunststoffen verwendet. Im österreichischen StraBen- und Bahnnetz existieren derzeit Tunnels auf einer Länge von ca. $650 \mathrm{~km}$, wobei die meisten Tunnels mit Drainagen ausgerüstet sind. Alleine bei den ÖBB ergeben sich somit aktuell ca. $445 \mathrm{~km}$ Drainagen. 2027 werden es bedingt durch die aktuelle Bautätigkeit ca. $1081 \mathrm{~km}$ sein. Zusammen mit den Tunnelbauwerken der ASFINAG und der Bundesländer sind in Österreich zum aktuellen Zeitpunkt weit über $1000 \mathrm{~km}$ Tunneldrainagen verbaut. Entweder aus geogenen Gründen und/oder aufgrund von beim Bau eingesetzten Baumaterialien kommt es in den Drainagen zu mineralischen Ausfällungen, die Versinterungen genannt werden. Zur Sicherstellung der Funktionstüchtigkeit der Bauwerke müssen daher diese Drainagen in regelmäßigen Abständen gewartet und gereinigt werden. Vor allem bei schwer lösbaren Versinterungen kann sich der Aufwand für die Reinigung von Drainagerohren exorbitant erhöhen, wodurch es nicht nur zu höheren Kosten in der Erhaltung, sondern neben einer möglichen Schädigung der Drainagen auch zu einer reduzierten Verfügbarkeit von Tunnelstrecken kommt. Aus diesem Grund wird versucht Methoden zu finden, mit denen es möglich ist Versinterungen zu vermindern, oder im Idealfall komplett zu verhindern.

\section{Versinterung von Drainagerohren}

\subsection{Entstehung \& Beschaffenheit von Versinte- rungen in Drainagerohren}

Versinterungen haben ihren Ursprung in komplexen physikochemischen Gesamtsystemen und etwaige Ursachenbehebungen stellen eine vielschichtige Herausforderung dar. Die Bildung von Karbonatversinterungen beruht zum einen auf der Entgasung oder auch Absorption von $\mathrm{CO}_{2}$ aus bzw. in die Drainagewässer, andererseits jedoch auch auf der mineralogischen Zusammensetzung und alkalischen Eigenschaft des Betons im Bauwerk, wobei hier im Wesentlichen die Auflösung des Minerals Portlandit $\left(\mathrm{Ca}(\mathrm{OH})_{2}\right)$ im Spritzbeton eine wichtige Rolle spielt [1-4]. Intensiver Kontakt von Wasser mit zementösen Materialien verändert die Eigenschaften und den Chemismus des Wassers wesentlich, wodurch alkalische, zumeist kalziumreiche Wässer generiert werden, welche zu einem erhöhten Versinterungspotential in den Tunneldrainagen führen können [2]. Des Weiteren hat auch die Geologie und die mineralogische Zusammensetzung der Gesteine im Aquifer (Grundwasserleiter) Auswirkungen auf den Grundwasserchemismus und somit auf das mögliche Versinterungspotential im Tunnel [5]. Zusätzlich beeinflusst die Geohydraulik den Weg des Wassers im Untergrund und damit die Kontaktzeit zum Gestein im Aquifer. Je intensiver diese Kontaktzeit (z. B. bei geringer Schüttung), desto größer ist der Einfluss auf den Wasserchemismus. Neben der Geohydraulik spielen in den Drainagen auch die Mischwasserbildungen und Strömungseigenschaften (turbulentes vs. Iaminares Fließen vs. stagnierende Bedingungen) eine wichtige Rolle [1, 2]. Die Versinterungen selbst können chemisch und petrographisch sehr komplex aufgebaut sein. Kalzit (Kalziumkarbonat $\mathrm{CaCO}_{3}$ ) ist zumeist die Hauptkomponente und kommt in einer groBen Vielfalt an Erscheinungsformen und mit verschiedener Konsistenz vor. Hinzu kommt noch das Vorhandensein weiterer Minerale als Nebenkomponenten, wie beispielsweise Aragonit ( $\mathrm{CaCO} 3$ Modifikation), Brucit $(\mathrm{Mg}(\mathrm{OH}) 2)$, Eisenoxide/Hydroxide (z. B. Goethit $\mathrm{FeO}(\mathrm{OH})$ und Hämatit $\mathrm{Fe}_{2} \mathrm{O}_{3}$ ) oder detritische Minerale (z. B. vielfältige Silikate), wodurch zur chemischen auch noch eine mineralogische Variabilität hinzukommt [1, 2]. Je nach Zusammensetzung und weiteren Randbedingungen der Ablagerung, können dadurch hinsichtlich Materialkonsistenz sehr weiche und schlammartige oder auch sehr harte, plattenartige Versinterungen entstehen (Abb. 1).

\subsection{Reinigung von Drainagerohren}

Um die Funktionalität des Tunnelbauwerks aufrechterhalten zu können, müssen die Drainagen regelmäßig gereinigt werden. Da eine Reinigung die Sperre des Gleises, der Richtungsfahrbahn oder gar des Tunnels bedeutet, müssen Reinigungsvorgänge möglichst effizient und schnell vonstattengehen. Gemäß der Systematik in der Richtlinie „Tunnelentwässerung“ der Österreichischen Vereinigung für Beton- und Bautechnik [6] gibt es prinzipiell mehrere Arten der Reinigung von Drainagerohren. Je nach Beschaffenheit der Versinterung können entweder hydraulische (Hochdruckspülung), hydromechanische (z.B. Vibrationsdüse), oder mechanische (Kettenschleuder, Fräser, Schlagbohrfräser) Reinigungsverfahren eingesetzt werden.

Bei sehr intensiven Reinigungsprozessen, v. a. wenn diese eine mechanische Komponente beinhalten, kann es dazu kommen, dass die Oberflächen der verbauten Drainagerohre angegriffen oder sogar zerstört werden (Abb. 2). Diese Art der Schädigung ist vor allem bei Rohren eine Gefahr, die vor einer Regulierung durch die Richtlinie Tunnelentwässerung 2003 [7], bzw. 2010 [6] eingebaut wurden [8].

Im Fall von Drainageleitungen in Tunnelbauwerken ist dies als besonders kritisch zu sehen, da diese aufgrund ihrer Situierung im Bauwerk nicht einfach ersetzt werden können. Um beschädigte Wasserleitungen austauschen zu können, müssten Teile der ganzen Tunnelwand inklusive Abdichtung und Sickerpackung entfernt werden, da es derzeit noch keine grabenlosen Sanierungsmethoden gibt, die nachweislich für die speziellen Anforderungen in Tunnelbauwerken (v.a. im Hinblick auf exzessive Reinigungsvorgänge mittels mechanischer Methoden, kontinuierlichen Wasserzutritt während der Sanierungsarbeiten, Drainageschlitze) geeignet sind. Dadurch ergibt sich abseits der Instandhaltungskosten ein weiterer Punkt, aufgrund dessen 

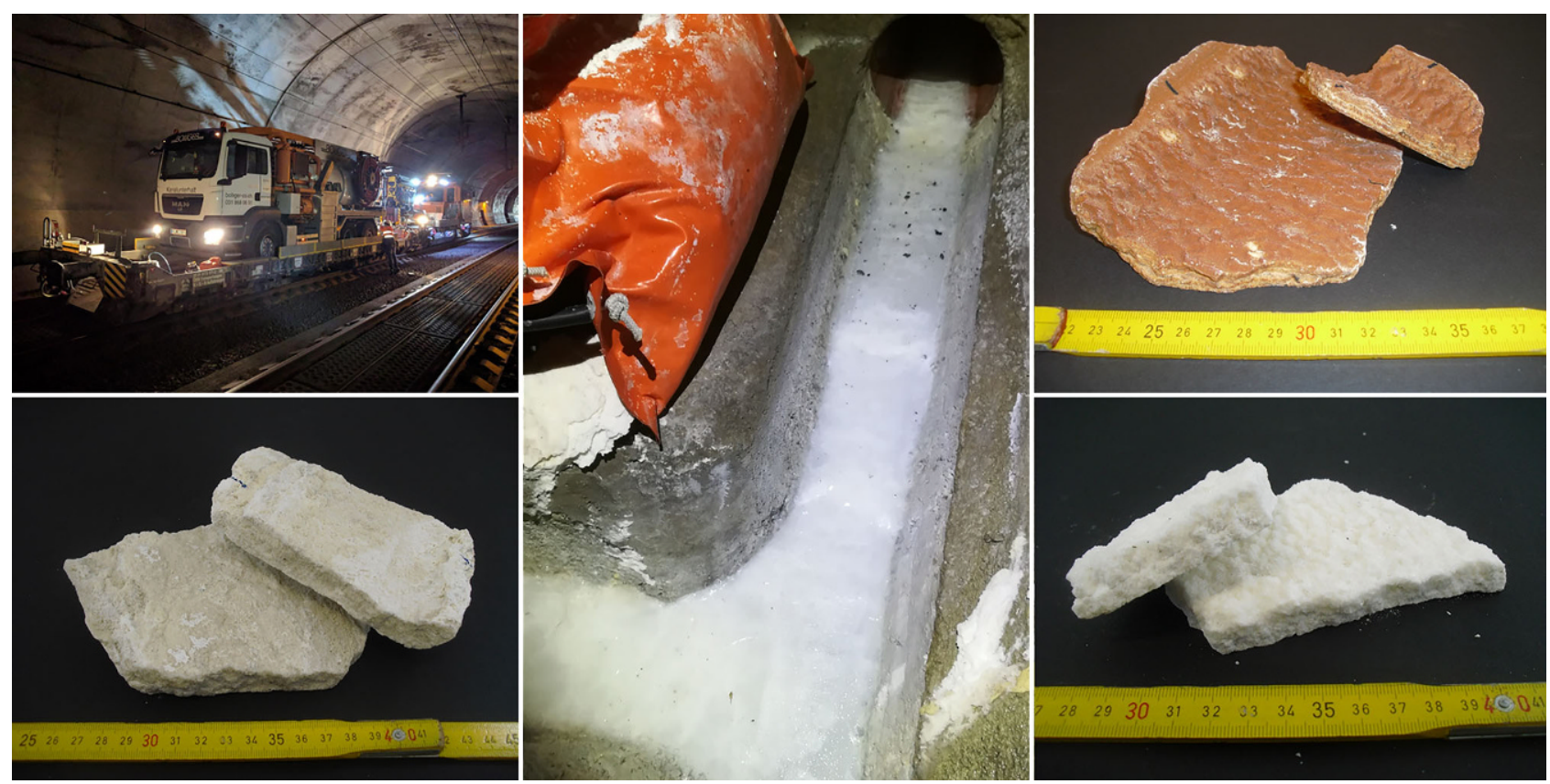

Abb. 1: Entfernung von Versinterungen in einem Eisenbahntunnel und die unterschiedliche Beschaffenheit (z. B. chemische Zusammensetzung, Färbung, Konsistenz) von Ablagerungen in Tunneldrainagen
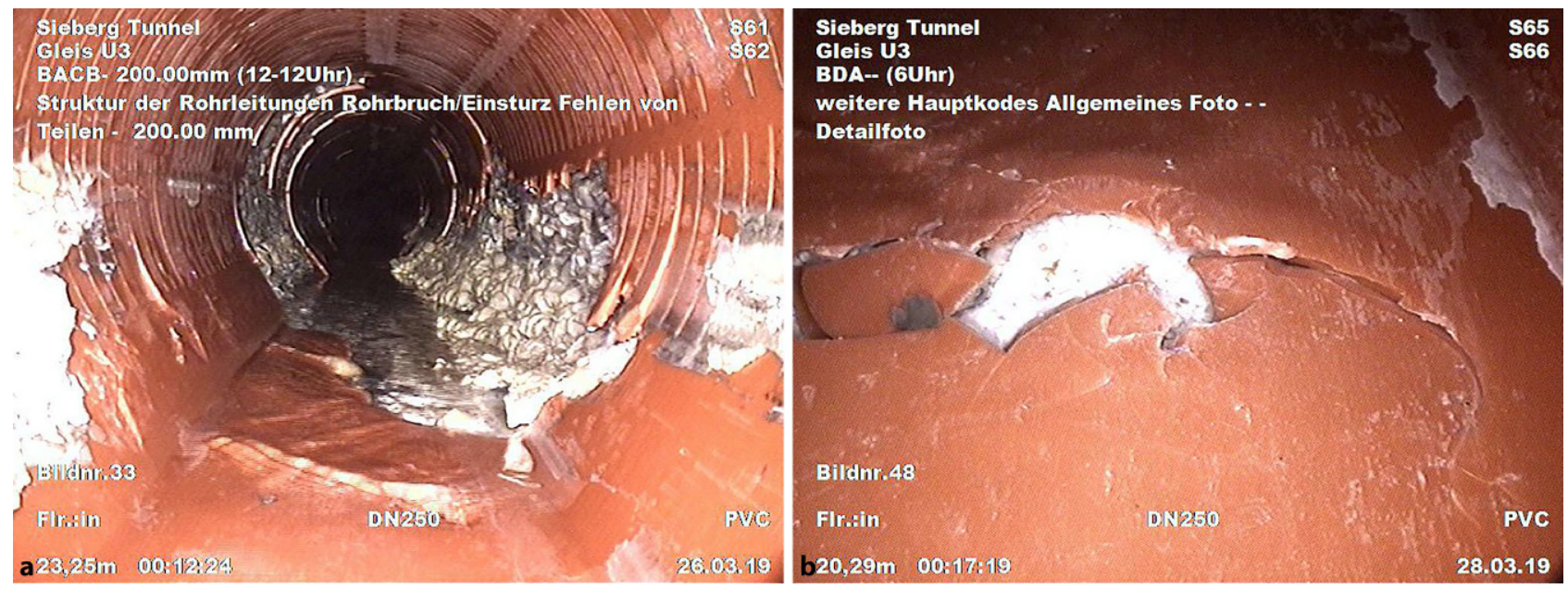

Abb. 2: Kamerabefahrung nach intensiven Reinigungsvorgängen im Tunnel Sieberg - komplette Zerstörung der Rohrwand (a), bzw. lokale Zerstörung der Drainagensohle nach intensiver mechanischer Reinigung (b)

eine Verminderung von Versinterungen in Drainagerohren dringend angestrebt wird.

Aufgrund der Dringlichkeit der gegenständlichen Thematik wird seit einigen Jahren intensiv nach Möglichkeiten zur Verringerung von Versinterungen in Tunnelbauwerken gesucht [9-13]. Als Unterstützung zur Reinigung kommen bei verschiedenen Tunnels derzeit "Härtestabilisatoren“ zum Einsatz. Darunter werden gering dosierte Zugaben verschiedener chemischer Wirkstoffe in fester und flüssiger Form zum Drainagewasser verstanden [12-16], welche entweder vorhandene Versinterungen reduzieren, die Ausfällung von Versinterungen verlangsamen, hemmen oder verhindern sollen. Diese bestehen meist aus verschiedenen Anteilen und molekularen Formen von Polyasparaginsäure oder anderen sogenannten "Grünen Inhibitoren“ (ökologisch unbedenkliche, biologisch gut abbaubare Karbonsäuren) oder auch anorganischen Säuren (konventionelle Inhibitoren; meist Phosphor-haltig). Abhängig vom verwendeten Wirkstoff und dessen Dosierung und zahlreichen - meist schwierig nachvollziehbaren natürlichen und technischen Randbedingungen - zeigen sich unterschiedliche Resultate, die von hoher bis zu praktisch nicht nachweisbarer Wirksamkeit reichen. Im Lainzer Tunnel konnten mittels verdünnter Salzsäure zum Beispiel gute Erfolge erzielt werden [16]. Ein Nachteil dieser Methode ist jedoch, dass die geltenden Umweltbestimmungen einen hohen Aufwand hinsichtlich Kontrolle und Dokumentation der Veränderung des Wasserchemismus bedingen. Zusätzlich wirken flüs- 


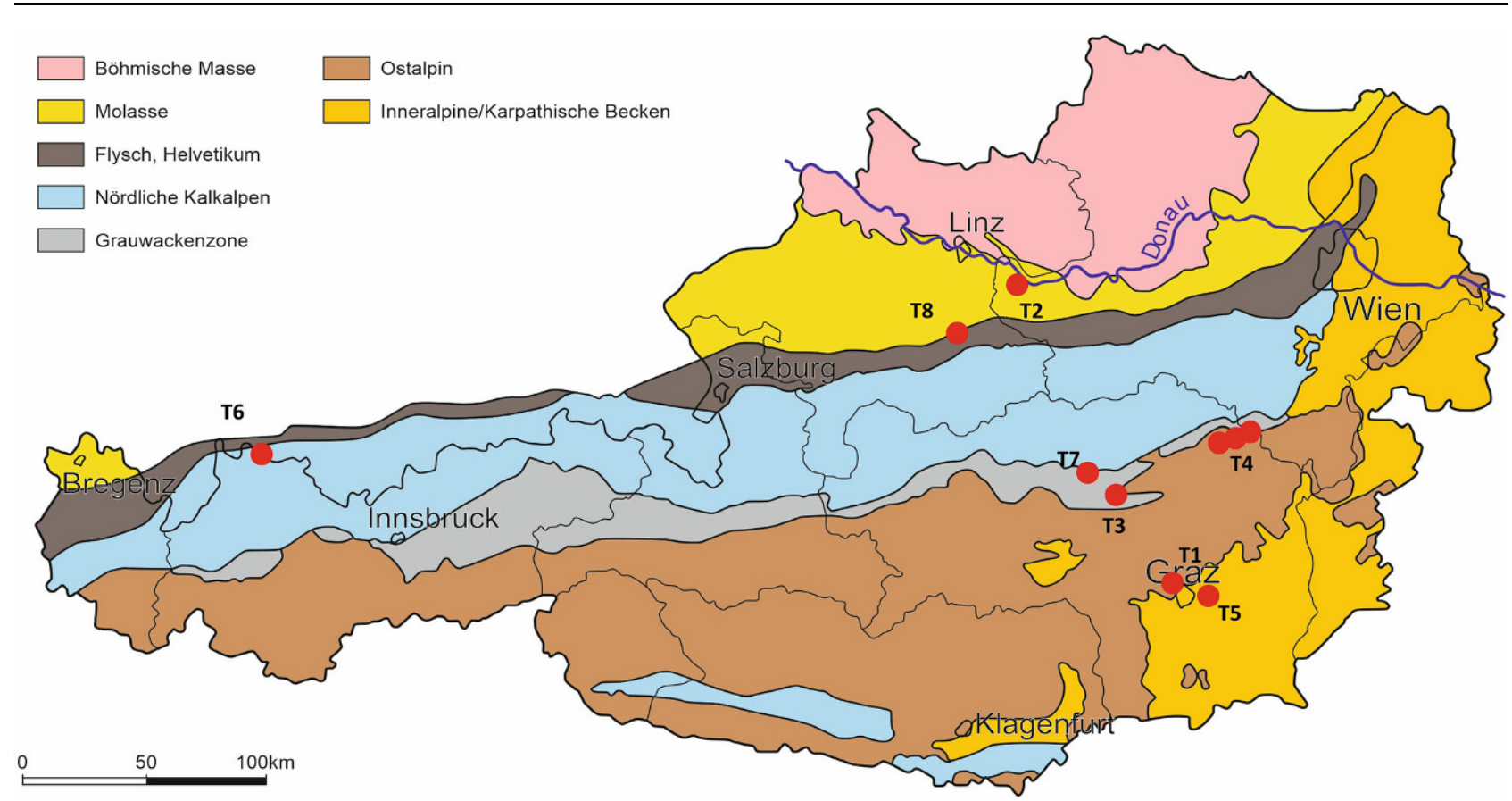

Abb. 3: Übersichtskarte erhobener Tunnel bezogen auf die großgeologischen Einheiten Österreichs nach [17] (T1-Plabutsch Tunnel, T2-Siebergtunnel, T3-Galgenbergtunnel,T4-Semmering Tunnelund TunnelSteinhaus, T5-TunnelHimmelreich, T6-GrenztunnelFüssen, T7-Zentrum am Berg, T8-Grünburg Tunnel)

sige Härtestabilisatoren derzeit nur in der Rohrsohle und nicht im Bereich der Drainageschlitze.

\section{Projekt PolyDrain}

Ausgehend von den beschriebenen Erfahrungen, wurde im Jahr 2017 ein von der ÖBB Infrastruktur AG, ASFINAG und BMVIT gemeinsam getragenes Forschungsprojekt gestartet, um sich mit dem Material der Drainagerohre selbst zu beschäftigen. Das primäre Ziel des ins Leben gerufenen Projekts „PolyDrain“ ist die Weiterentwicklung der Kunststoffe, die für die Produktion von Drainagerohren verwendet werden, um eine verringerte Versinterungsneigung zu erzielen. Zur Bearbeitung dieser komplexen und interdisziplinären Fragestellung wurden Experten aus den Fachrichtungen Tunnelbau, Geologie und Hydrogeologie, Hydrochemie und Mineralogie, Materialwissenschaften und Polymerchemie zusammengezogen.

\subsection{Entwicklung, Methodik \& Material}

\subsubsection{Ursachenforschung und Erhebung}

Bevor Kunststoffmaterialien gezielt in Richtung Versinterungsneigung modifiziert werden können, ist es wichtig, die vorherrschenden Mechanismen der Sinterbildung selbst zu verstehen. Da diese, wie bereits beschrieben, von sehr vielen Faktoren beeinflusst werden können, wurde in einem ersten Schritt eine Erhebung bestehender Tunnelbauwerke in Österreich inklusive Bewertung des vorherrschenden Versinterungspotentials durchgeführt. (Abb. 3) Basierend auf dieser Datenmenge sollen kritische Faktoren, die die Versinterungsneigung in realen Bauwerken maßgeblich beeinflussen, ausfindig gemacht werden. Um diese für die Ursachenforschung notwendigen Daten verwertbar zu machen, wurden sie in einer übersichtlichen Datenbank zusammengefasst, mit der es möglich ist, je nach Bedarf die Randbedingungen in verschiedenen Bauwerken zu erheben.

\subsubsection{Materialentwicklung}

Zur Optimierung der Eigenschaften verwendeter Kunststoffe in Drainagerohren stehen zwei Wege zur Verfügung. Entweder werden aktive Füllstoffe in den Kunststoff eingearbeitet, oder es werden die Oberflächen der Rohre selbst modifiziert. Beide Arten der Modifizierung haben nachweislich Vor- und Nachteile. Durch die Verwendung von Füllstoffen wird der aktive Bestandteil der Mischung in der gesamten Rohrwand verteilt. Für eine Verringerung der Versinterungsneigung wird dieser jedoch primär an der Rohrinnenwand benötigt. Daher muss bei diesem Verfahren mehr Wirkstoff eingebracht werden, um die gleiche Effizienz wie bei einem Verfahren zur gezielten Modifizierung der Oberfläche zu erreichen. Sollte es jedoch trotz der Verminderung von Versinterungen notwendig sein, die Drainagen später zu spülen, könnte es sein, dass die modifizierte Oberfläche beschädigt oder gar komplett abgetragen wird (Abb. 4), wodurch keine Wirksamkeit gegen das Aufwachsen von Versinterungen mehr gegeben ist.

Aufgrund der mechanischen Reinigungsmethoden, welche derzeit für Tunneldrainagen verwendet werden und nachweislich Oberflächen angreifen können, ist daher der Weg des Einmischens von Füllstoffen in puncto Sicher- 
Abb. 4: Möglichkeiten zur Modifizierung von Drainagerohrmaterialien mit (a) aktiven Füllstoffen, oder (b) Oberflächenbeschichtung

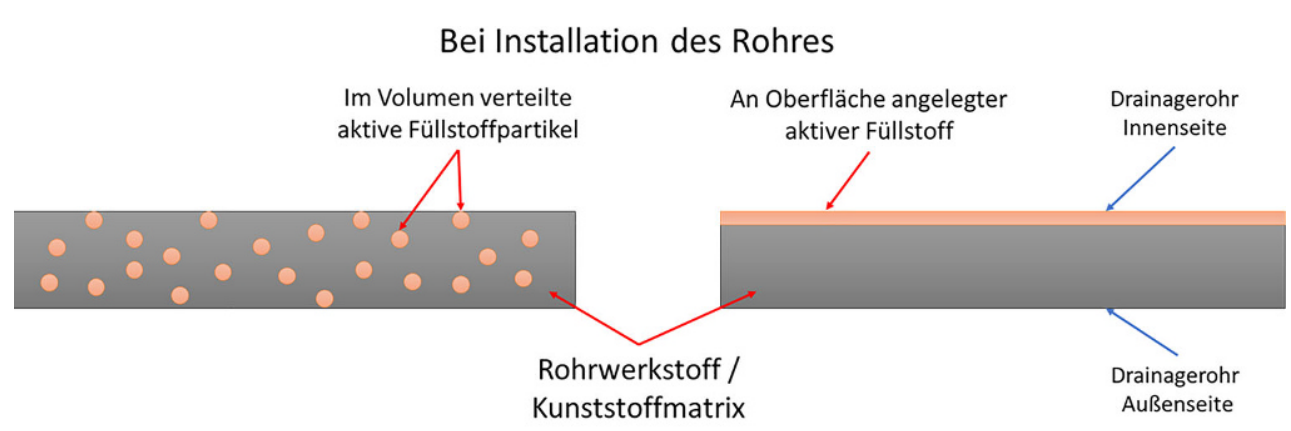

Nach intensivem Reinigungsprozess
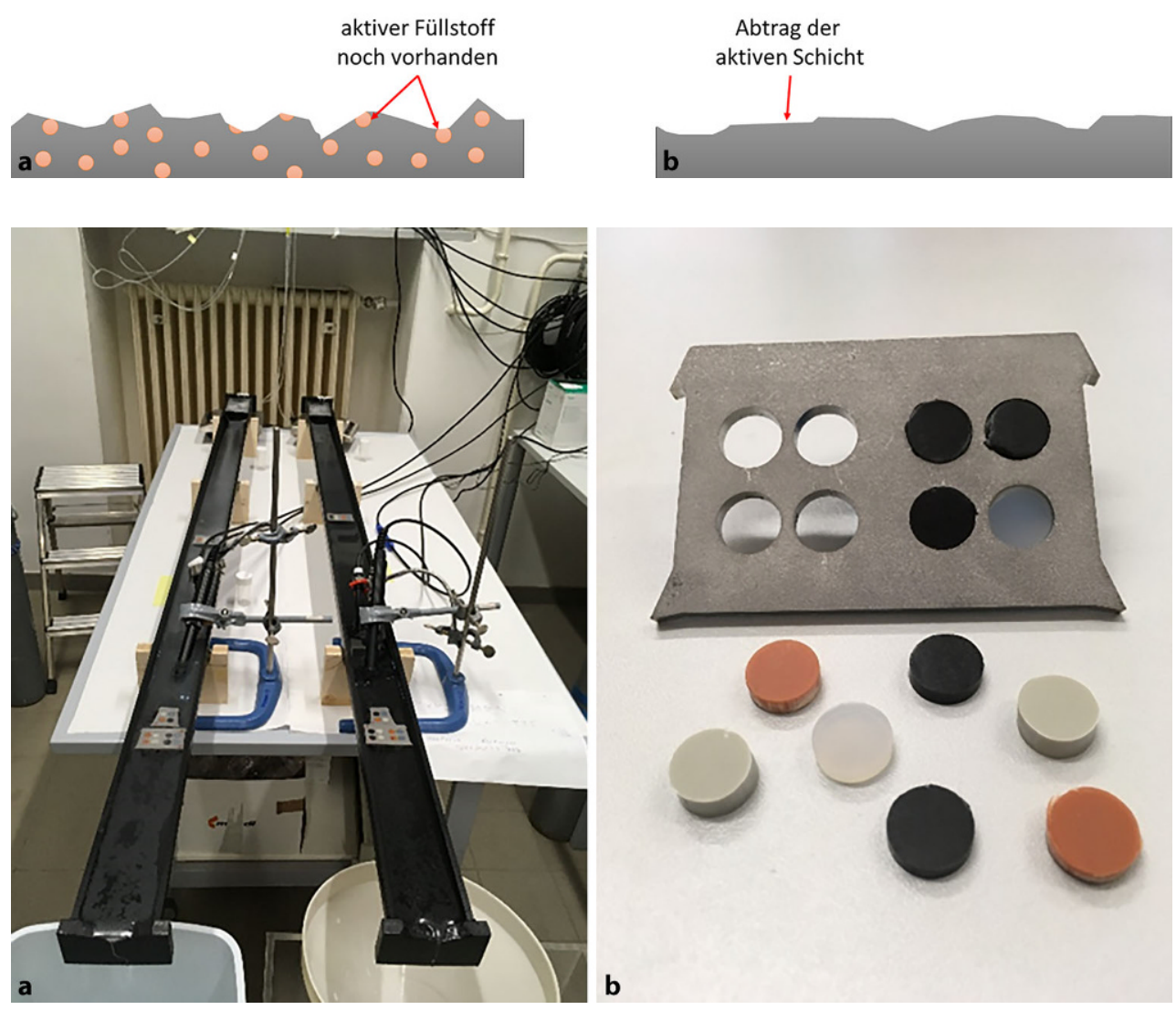

Abb. 5: Entwicklung des a Versinterungsprüfstands und $\mathbf{b}$ die dafür entwickelten Probenträger zur Auslagerung modifizierter Kunststoffproben heit als vorteilhaft anzusehen. Zusätzlich ist zu beachten, dass die Drainageschlitze in Drainagerohren erst nach der Herstellung des Rohres selbst eingebracht werden. Dies würde bei rein Oberflächen-modifizierten Drainagerohren dazu führen, dass im Bereich der Drainageschlitze keine aktiven Wirkstoffe vorhanden sind. Um die Wirksamkeit der Füllstoffe in einer Kunststoffmatrix zu prüfen, wurden diese in ein kommerziell erhältliches Material (Polyethylen) eingemischt und zu Platten geformt. Aus diesen Platten wurden zylindrische Probekörper mit einem Durchmesser von $10 \mathrm{~mm}$ für weitere Untersuchungen entnommen.

Zur Bestimmung der Wirksamkeit der verwendeten Füllstoffe ist ein geeignetes Prüfverfahren inklusive Beurteilungsschema notwendig. Daher wurde ein Prüfstand entwickelt, mit dem es möglich ist, die Abscheidung von Kalziumkarbonat-Mineralen aus einer an diesen Mineralen übersät- tigten Lösung im Labormaßstab nachzustellen. Der Fokus der Untersuchungen liegt auf der vielfältigen Kristallisation von Kalziumkarbonat-Mineralen an der Oberfläche der Probekörper. Die verschiedenen zu testenden Kunststoffproben werden jeweils am Anfang und am Ende von zwei parallel aufgebauten Fließstrecken in speziell hierfür angefertigten Halterungen (Abb. 5) in die Lösung eingebracht. Letztere erlauben eine Differenzierung der mineralischen Ablagerung zwischen Mineral-Aufwachsen auf dem Kunststoffsubstrat und der Sedimentation von KarbonatkristalIen in Suspension (heterogene versus homogene Kristallnukleation). Während die Abscheidungen an der Oberseite der individuellen Kunststoffe in der Lösung gänzlich oder teilweise durch Mineral-Sedimentation durch zuvor in Suspension befindliche Kristalle entstanden sein könnten, sind die gebildeten Präzipitate auf der Unterseite der individuel- 


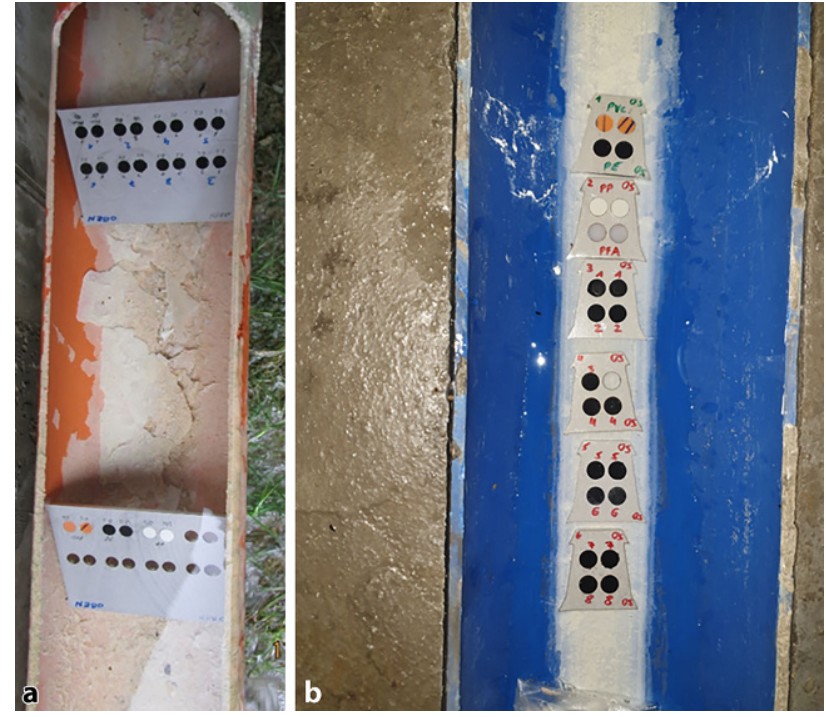

Abb. 6: Auslagerung modifizierter Kunststoffe unter realen Bedingungen $\mathbf{a}$ am Drainageleitungsausgang des Tunnel Steinhaus und $\mathbf{b}$ in der Drainageleitung des Lainzer Tunnels

Ien Kunststoffe ausschließlich an den einzelnen Kunststoffen aufgewachsen. Die Beurteilung des variablen Abscheidungsverhaltens von Mineralen und besonders von Karbonaten und deren Bildungsrate erfolgt weiters über hydrochemische Computer-Modellierungen. Die auf den individuellen Kunststoffen gebildeten Abscheidungen werden mineralogisch und chemisch charakterisiert und mittels bildgebenden Verfahren, wie räumlich hochauflösende elektronenoptische Analytik (z.B. Rasterelektronenmikroskop), untersucht.

Zur Absicherung der Aussagekraft dieser im Labor durchgeführten Versuche werden gleichzeitig auch Proben in realen Tunnelbauwerken ausgelagert. Dies dient in erster Linie zur Validierung der Ergebnisse aus dem Labor und zur Untersuchung der Auswirkung verschiedener Bergwassermischungen auf die individuelle Wirksamkeit der gewählten Füllstoffe. Dazu werden derzeit Proben sowohl in einem eigens konzipierten Prüfstand am Drainagenausgang des Tunnels Steinhaus (vgl. Abb. 6a), sowie in den Drainagen des Lainzer Tunnels (vgl. Abb. 6b) ausgelagert. Sobald Versinterungen auf den im Drainagewasser eingelagerten Probekörpern mit freiem Auge sichtbar sind, werden diese entnommen und analog zu den Kunststoffsubstraten im Labor (vgl. Abb. 5b) untersucht.

\subsection{Erste Ergebnisse}

Nach der Entnahme der Kunststoff-Probekörper aus dem Prüfstand bzw. den realen Bauwerken werden diese mittels unterschiedlicher Methoden analysiert. Zum einen wird, wie in Abb. 7 dargestellt, untersucht, wie viele Kristalle (Kalzit, Aragonit) auf den jeweiligen Substraten aufgewachsen sind. Dies dient zur quantitativen Beurteilung der Wechselwirkung der gewählten Füllstoffe. Zusätzlich werden die aufgewachsenen karbonatischen Kristalle mittels Rasterelektronenmikroskop im Detail untersucht. Dies dient primär zur qualitativen Beurteilung der Kristallstruktur und Verwachsung der Versinterung. Bisherige Untersuchungen haben gezeigt, dass auch diese Versinterungseigenschaften von dem als Substrat gewählten Kunststoff selbst wesentlich beeinflusst werden können.

Im Projekt PolyDrain werden derzeit sieben verschiedene Materialmischungen hinsichtlich ihrer Wechselwirkungen und Wirksamkeit gegen Versinterung untersucht. Wie in Abbildung Abb. 7 zu sehen, ist es mittels der Zugabe von Füllstoffen möglich, das Versinterungsverhalten des jeweiligen Matrixwerkstoffes (a) signifikant zu beeinflussen. Je nach gewählten Füllstoffen kann eine starke Verringerung (b), Vermehrung oder gar Änderung der Morphologie (c) der aufwachsenden Kristalle erreicht werden. Diese ersten Ergebnisse lassen darauf schließen, dass der gewählte Ansatz der Modifizierung des Matrixwerkstoffes großes Potential für die Entwicklung von versinterungsoptimierten Drainagerohren aus Kunststoff hat. Bevor diese Wirkstoffe jedoch im großtechnischen Maßstab eingesetzt werden können, benötigt es weiterer Validierungsund Langzeitversuche, sowohl im Labor, als auch in realen Bauwerken.

\section{Zusammenfassung und Ausblick}

Ausgehend von der Problemstellung aufwändig zu reinigender Tunneldrainagen wurde das interdisziplinäre Forschungsprojekt „PolyDrain“ initiiert. Neben der Erhebung kritischer Tunnelbauwerke wird vor allem an der Entwicklung versinterungsarmer Kunststoffmaterialmischungen geforscht. Erste Ergebnisse im Tunnel und im Labor haben bereits gezeigt, dass es möglich ist, das Versinterungsverhalten von Kunststoffmaterialien signifikant und vorteilhaft zu verändern. Ausgehend von diesen Ergebnissen werden derzeit Validierungsversuche an Kunststoffproben, die in
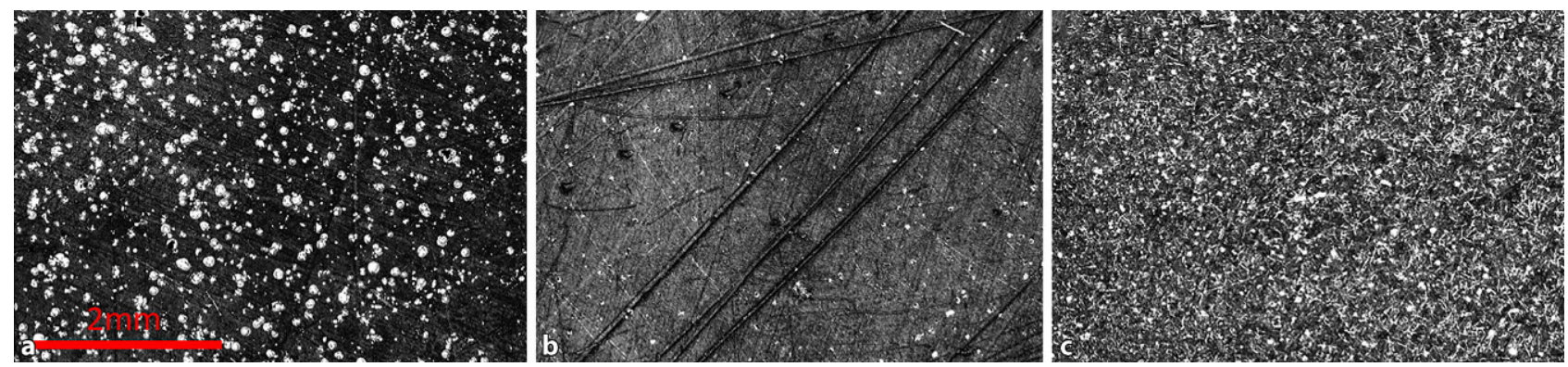

Abb. 7: Veränderung der Quantität (b) und Morphologie (c) von aufwachsenden Kalzit-Kristallen im Vergleich zu reinem Polyethylen (a) 
realen Tunnelbauwerken ausgelagert sind, durchgeführt. Zusätzlich sind Langzeituntersuchungen geplant, um die Wechselwirkungen und Wirksamkeit hinsichtlich Versinterung auch auf längere Zeit betrachtet zu untersuchen. Unter der Voraussetzung einer positiven Langzeitevaluierung sollte es möglich sein, die Instandhaltungskosten zukünftiger Bauwerke deutlich zu senken.

Danksagung. Die durchgeführten Arbeiten in dieser Veröffentlichung wurden im Rahmen des VIF-2016 Projekts „PolyDrain“ (10549986) mit Beteiligung der ÖBB-Infrastruktur AG, sowie der ASFINAG und des Bundesministeriums für Verkehr, Innovation und Technologie durchgeführt. Besonderer Dank gilt allen weiteren beteiligten Projektpartnern des Projekts „PolyDrain“: TU-Graz, BOKU Wien, Montanuniversität Leoben, Ingenieurbüro Strobl und Polymer Competence Center Leoben $\mathrm{GmbH}$.

Funding. Open access funding provided by Montanuniversität Leoben.

Open Access Dieser Artikel wird unter der Creative Commons Namensnennung 4.0 International Lizenz (http://creativecommons.org/licenses/ by/4.0/deed.de) veröffentlicht, welche die Nutzung, Vervielfältigung, Bearbeitung, Verbreitung und Wiedergabe in jeglichem Medium und Format erlaubt, sofern Sie den/die ursprünglichen Autor(en) und die Quelle ordnungsgemäß nennen, einen Link zur Creative Commons Lizenz beifügen und angeben, ob Änderungen vorgenommen wurden.

\section{Literatur}

1. Dietzel, M.; Rinder, T.; Niedermayr, A.; Mittermayr, F.; Leis, A.; Klammer, D. et al.: Ursachen und Mechanismen der Versinterung von Tunneldrainagen. BHM Berg- und Hüttenmännische Monatshefte 153 (2008), H. 10, S. 369-372

2. Rinder, T.; Dietzel, M.; Leis, A.: Calcium carbonate scaling under alkaline conditions-Case studies and hydrochemical modelling. Applied Geochemistry 35 (2013), pp132-41. https://doi.org/10.1016/j. apgeochem.2013.03.019

3. Dietzel, M.; Rinder, T.; Leis, A.; Reichl, P.; Sellner, P.; Draschitz, C. et al.: Koralm Tunnel as a Case Study for Sinter Formation in Drainage Systems - Precipitation Mechanisms and Retaliatory Action. Geomechanik und Tunnelbau 1 (2008), iss. 4, pp 271-278. https:// doi.org/10.1002/geot.200800024

4. Dietzel, M.; Purgstaller, B.; Leis, A.; Reichl, P.; Stadler, H.; Niedermayr, A. et al.: Current challenges for scaling of tunnel drainage systems - Modelling approaches, monitoring tools and prevention strategies / Aktuelle Herausforderungen bei der Versinterung von Tunneldränagen - Modellierungsansätze, Monitoringwerkzeuge und Präventionsstrategien. Geomechanik und Tunnelbau 6 (2013), iss. 6, pp 743-753. https://doi.org/10.1002/geot.201310014

5. Wu Z, Cui Y, Barrett AG, Moreno MM, Deng Y. Role of surrounding soils and pore water in calcium carbonate precipitation in railway tunnel drainage system. Transportation Geotechnics 2019;21:100257. https://doi.org/10.1016/j.trgeo.2019.100257

6. Österreichische Vereinigung für Beton- und Bautechnik: Richtlinie Tunnelentwässerung. Wien, 2010

7. Österreichische Vereinigung für Beton- und Bautechnik: Ausbildung von Tunnelentwässerung. Wien, 2003

8. Schachinger, T.; Arbeiter, F.J.; Eichinger, S.; Saliger, F.: Research on pipe materials for tunnel drainage by the ÖBB Task Force Drainage. Geomechanik und Tunnelbau 12 (2019), iss. 5, pp 467-471 https:// doi.org/10.1002/geot.201900022

9. Schachinger, T.; Sperger, L.; Heissenberger, R.; Wagner, O.K.: Task Force Drainage (TFD)-The project for life after. Geomechanik und Tunnelbau 10 (2017), iss. 6, pp 779-787. https://doi.org/10.1002/geot. 201700045

10. Wagner, O.K.; Koch, D.; Lemmerer, J.; Druckfeuchter, H.; Petraschek, T.: Maintenance-optimised drainage system for the New Semmering Base tunnel and Pummersdorf Tunnel / Instandhaltungsoptimiertes Entwässerungssystem für den Semmering-Basistunnel neu und Tunnel Pummersdorf. Geomechanik und Tunnelbau 7 (2014), iss. 5, pp 626-635. https://doi.org/10.1002/geot.201400032

11. Schachinger, T.; Zagar, B.; Schwab, C.; Saliger, F; Stur, M.: Current research by ÖBB Infrastruktur AG on scale monitoring without track closures. Geomechanik und Tunnelbau 11 (2018), iss. 3, pp 277-285. https://doi.org/10.1002/geot.201800012

12. Zhou, Y.; Zhang, X.; Wei, L.; Liu, S.; Zhang, B.; Zhou, C.: Experimental Study on Prevention of Calcium Carbonate Crystallizing in Drainage Pipe of Tunnel Engineering. Advances in Civil Engineering, vol. 2018, Article ID 9430517, 11 p. https://doi.org/10.1155/2018/ 9430517

13. Girmscheid, G.; Gamisch, T.; Meinlschmidt, A.: Versinterung von Tunneldrainagen - Empfehlungen für die Instandhaltung von Tunneln. Bauingenieur 78 (2003), H. 12, S. 571-580

14. Galli, M.: Härtestabilisierung in kalkführenden Entwässerungen: Verhinderung von Kalkablagerungen. Verlags-AG der akademischen technischen Vereine, Schweizer Ingenieur und Architekt, 118 (2000), H. 12, S. 249-253

15. Vollmann, G.: Zur Härtestabilisierung als versinterungsreduzierende Maßnahme für Tunneldränagesysteme, 1. Aufl., Göttingen: Cuvillier, 2005

16. Stur, M.; Ottner, F.; Schachinger, T.; Wriessnig, K.: Lokale Beseitigung von Versinterungen in Bergwasserdrainagen. Tunnel 2015, https:// www.tunnel-online.info/de/artikel/tunnel_Lokale_Beseitigung_von_ Versinterungen_in_Bergwasserdrainagen_2242030.html

17. Wessely, G.; Liebl, W.: Oil and Gas in Alpidic Thrustbelts and Basins of Central and Eastern Europe. In: Wessely, G. (ed.): Petroleum Exploration and Production in Thrustbelts and Foreland Basins: 1994 EAGE Conference in Vienna. London: Geological Society, 1996, p. 217-235

Hinweis des Verlags. Der Verlag bleibt in Hinblick auf geografische Zuordnungen und Gebietsbezeichnungen in veröffentlichten Karten und Institutsadressen neutral. 